\title{
Performance of PSI, CURB-65, and SCAP scores in predicting the outcome of patients with community-acquired and healthcare-associated pneumonia
}

\author{
Marco Falcone $\cdot$ Salvatore Corrao • \\ Mario Venditti · Pietro Serra - Giuseppe Licata
}

Received: 27 September 2010/ Accepted: 5 January 2011/Published online: 20 January 2011

(C) SIMI 2011

\begin{abstract}
The objective was to compare three score systems, pneumonia severity index (PSI), the Confusion-UreaRespiratory Rate-Blood pressure-65 (CURB-65), and severe community-acquired pneumonia (SCAP), for prediction of the outcomes in a cohort of patients with communityacquired (CAP) and healthcare-associated pneumonia (HCAP). Large multi-center, prospective, observational study was conducted in 55 hospitals. HCAP patients were included in the high classes of CURB-65, PSI and SCAP scores have a mortality rate higher than that of CAP patients. HCAP patients included in the low class of the three severity rules have a significantly higher incidence of adverse events, including development of septic shock, transfer into an ICU, and death $(p<0.01)$. At multivariate Cox regression analysis, inclusion in the severe classes of PSI, CURB-65, or SCAP scores and receipt of an empirical therapy not adherent to international guidelines prove to be risk factors independently associated with poor outcome. PSI, CURB65 , and SCAP score have a good performance in patients with CAP but are less useful in patients with HCAP, especially in patients classified in the low-risk classes.
\end{abstract}

Keywords Community-acquired pneumonia . Healthcare-associated pneumonia · PSI · CURB 65

For the Italian Society of Internal Medicine (SIMI).

M. Falcone $(\square) \cdot$ M. Venditti · P. Serra

Department of Public Health and Infectious Diseases, Policlinico

Umberto I, University of Rome "La Sapienza", Rome, Italy

e-mail: marcofalc@libero.it

S. Corrao · G. Licata

Department of Internal Medicine, University of Palermo,

Palermo, Italy

\section{Introduction}

Traditionally, pneumonia occurring in patients living in the community has been categorized as community-acquired pneumonia (CAP). However, the designation of healthcareassociated pneumonia (HCAP) has been recently introduced to include a population of nursing-home residents, patients receiving home- or hospital-based intravenous therapy, undergoing dialysis, or with a history of recent hospitalization [1]. Patients with HCAP have a more severe disease with longer hospital stay and higher mortality rates $[1,2]$, and inclusion criteria for HCAP have been associated with an increased risk for multidrug-resistant (MDR) pathogens [3].

The two most widely used predictive score systems for CAP are the pneumonia severity index (PSI) [4] and the Confusion-Urea-Respiratory Rate-Blood pressure-65 (CURB-65) score [5]. Recently, a new clinical prediction rule for severe $\mathrm{CAP}$, the severe community-acquired pneumonia (SCAP) score, has been developed [6]. Few studies have examined their performance in predicting outcome of patients with HCAP [7].

The aim of this study was to compare PSI, CURB-65, and SCAP scores for prediction of outcomes in a cohort of patients with CAP and HCAP.

\section{Methods}

Setting and patients

This cohort study was performed prospectively in 59 divisions of internal medicine in 55 Italian hospitals [1]. An institutional review board of the Italian Society of Internal Medicine (SIMI) approved the study. We classified 
patients as having HCAP if they had attended a hospital or hemodialysis clinic or received intravenous chemotherapy in the past 30 days, had been admitted to an acute-care hospital for at least 2 days or had surgery in the past 180 days, or resided in a nursing home or long-term care facility. CAP was defined as a diagnosis of pneumonia in patients living in the community who did not meet any of the criteria for HCAP. We stratified patients into risk classes using the PSI [4], CURB-65 [5], and SCAP scores [6]. This analysis was not designed in the original study protocol, but is a post hoc analysis using prospectively collected data. At the clinical end points, we retrieved the variables of septic shock, need for transfer into an intensive care unit (ICU), and in-hospital death.

Antimicrobial treatment evaluation

We defined empirical antibiotic therapy as antibiotics administered on the first day of therapy for pneumonia. We considered the antibiotic regimen as adherent to guidelines if it was concordant with the available American Thoracic Society and Infectious Diseases Society of America guidelines for CAP and HCAP [8, 9]

\section{Statistical analysis}

Data are showed as mean [95\% Confidence Intervals (CI)] for quantitative variables and as relative frequencies $(95 \%$ CI) for categorical variables. We performed nonparametric tests for group comparison and the generalized Fisher exact test for contingency table analysis.

The Cox regression analysis was performed to find the model that best predicted in-hospital death or the combined outcome (septic shock, need of transfer into an ICU, and in-hospital death) using fractional polynomial regression to study relationships between independent quantitative variables and outcome. After univariate analysis, independent variables at $p$ value level of 0.20 were chosen and included in the model with an afterward selection process. Clinical variables already included in the three severity rules (PSI, CURB-65, and SCAP score) were not further considered. Finally, a cluster procedure was used to validate the best-fit regression model and adjust standard errors for intragroup correlation. Hazard ratios and their 95\% CI were computed. STATA/SE, version 9.2 for Windows (Stata Corp, College Station, TX) was used to analyze the data.

\section{Results}

The sample included 313 patients with pneumonia, 223 (71.2\%) classified as having a CAP and 90 (28.8\%) as having a HCAP. The two groups did not significantly differ in terms of mean age, gender distribution, and presence of comorbidities [1]. Table 1 summarizes some baseline characteristics of patients with CAP and HCAP included in the study. When compared to patients with CAP, HCAP patients were less frequently included in low-risk classes of PSI (23.3 vs $8.9 \%, p=0.004$ ), CURB-65 (43.9 vs $34.4 \%$, $p=0.01)$, and SCAP score (41.7 vs $23.3 \%, p=0.004)$, and less frequently treated with an empirical therapy that was consistent with available guidelines (26.7 vs. $58.7 \%$, $p<0.001)$. The overall mortality rate was $9.9 \%$, and was significantly higher in patients with HCAP $(17.8 \%)$ than with CAP (17.8 vs. $6.7 \%, p<0.01)$.

Differences in the proportion of in-hospital mortality in each severity class, as assessed by PSI, CURB-65 and SCAP score, are presented in Table 2. HCAP patients included in the low class of the three severity rules had

Table 1 Baseline clinical characteristics of patients with CAP and HCAP

\begin{tabular}{lclc}
\hline & CAP & HCAP & $P$ \\
& $n=223$ & $n=90$ & \\
\hline Respiratory rate $>30$ breaths/min & $73(32.8 \%)$ & $31(34.4 \%)$ & 0.7 \\
Systolic blood pressure $<90 \mathrm{mmHg}$ & $6(2.7 \%)$ & $6(6.7 \%)$ & 0.1 \\
Pulse $>125 / \mathrm{min}$ & $73(32.7 \%)$ & $35(38.9 \%)$ & 0.2 \\
Altered mental status & $44(19.8 \%)$ & $24(26.7 \%)$ & 0.3 \\
$\mathrm{Blood}$ urea nitrogen $>30 \mathrm{mg} / \mathrm{dl}$ & $69(30.9 \%)$ & $40(44.4 \%)$ & $\mathbf{0 . 0 2}$ \\
$\mathrm{PaO}_{2} / \mathrm{FiO}{ }_{2}<300$ & $59(26.5 \%)$ & $38(42.2 \%)$ & $\mathbf{0 . 0 1}$ \\
$\mathrm{Bilirubin}>1.5 \mathrm{mg} / \mathrm{dL}$ & $26(11.7 \%)$ & $13(14.4 \%)$ & 0.5 \\
Hematocrit $>30 \%$ & $95(42.6 \%)$ & $60(66.7 \%)$ & $<\mathbf{0 . 0 1}$ \\
Glucose $>250 \mathrm{mg} / \mathrm{dl}$ & $34(15.2 \%)$ & $22(24.4 \%)$ & 0.07 \\
\hline Bld & & &
\end{tabular}

Bold values are those statistically significant

Table 2 In-hospital mortality in each severity class assessed by PSI, CURB-65 and SCAP scores in patients with CAP and HCAP

\begin{tabular}{llll}
\hline & CAP & HCAP & $P$ \\
\hline PSI class & & & \\
$\quad$ Low (I) & 0 & $12.5(0-36.6)$ & 0.133 \\
$\quad$ Intermediate (II) & $4.6(0.6-8.5)$ & $11.1(3.9-18.3)$ & 0.157 \\
$\quad$ High (III) & $16.1(7.7-24.6)$ & $27(13.9-40.1)$ & 0.206 \\
CURB-65 Class & & & \\
$\quad$ Low (I) & $3.1(0-7.1)$ & $12.9(2.6 .-23.2)$ & 0.057 \\
Intermediate (II) & $10(3.9-16.1)$ & $12.5(1.8-23.2)$ & 0.742 \\
$\quad$ High (III) & $8.6(0-18.6)$ & $29.6(12.2-47)$ & $\mathbf{0 . 0 4 5}$ \\
SCAP Class & & & \\
$\quad$ Low (I) & $2.1(0-5.8)$ & $9.5(0-20.8)$ & 0.154 \\
Intermediate (II) & $8.7(2.8-14.7)$ & $12.2(3-21.4)$ & 0.538 \\
High (III) & $12(1.7-22.3)$ & $32.1(16-48-2)$ & $\mathbf{0 . 0 3 9}$ \\
\hline
\end{tabular}

Data are shown as percentage $(95 \% \mathrm{CI})$

Bold values are those statistically significant 
higher mortality rates (range $9.5-12.9 \%$ ) than CAP ones (range $0-3.1 \%$ ).

Outcome of HCAP and CAP patients was also evaluated in terms of cumulative incidence of adverse events, including development of septic shock, transfer into an ICU, and death. As described in Table 3, the incidence of adverse events is significantly higher among HCAP patients included in the low class of PSI $(p=0.01)$, CURB-65 $(p=0.01)$, and SCAP score $(p=0.04)$. Among HCAP patients, inclusion in the low-risk classes is not associated with higher percentages of empirical antibiotic therapy not adherent to international guidelines (data not shown).

Results of the multivariate Cox regression analysis are described in Tables 4 and 5. Inclusion in the severe classes of PSI, CURB-65, and SCAP scores and receipt of an empirical therapy not adherent to international guidelines prove to be risk factors independently associated with intra-hospital mortality or development of adverse events.

Table 3 Cumulative incidence of adverse events (septic shock, ICU transfer, and death) in each severity class assessed by PSI, CURB-65, and SCAP scores in patients with CAP and HCAP

\begin{tabular}{llll}
\hline & CAP & HCAP & $P$ \\
\hline PSI class & & & \\
$\quad$ Low (I) & 0 & $25(0-51.9)$ & $\mathbf{0 . 0 1 6}$ \\
Intermediate (II) & $7.3(2.8-11.9)$ & $15.5(7.1-24)$ & 0.139 \\
High (III) & $21(12.4-29.5)$ & $29.7(16.6-42.8)$ & 0.342 \\
CURB-65 Class & & & \\
Low (I) & $3.1(0-7.1)$ & $16.1(5.2 .-27.1)$ & $\mathbf{0 . 0 1 9}$ \\
Intermediate (II) & $11.1(5-17.2)$ & $15.6(3.6-27.7)$ & 0.536 \\
High (III) & $22.9(7.1-38.7)$ & $37(18.8-55.2)$ & 0.267 \\
SCAP Class & & & \\
Low (I) & $2.1(0-5.8)$ & $14.3(1.6-26.9)$ & $\mathbf{0 . 0 4 3}$ \\
Intermediate (II) & $10(3.7-16.3)$ & $14.6(4.5-24.8)$ & 0.550 \\
High (III) & $22(9.9-34.1)$ & $39.3(23-55.5)$ & 0.122 \\
\hline
\end{tabular}

Data are shown as percentage $(95 \% \mathrm{CI})$

Bold values are those statistically significant

\section{Discussion}

This study has evaluated the performance of three severity rules, PSI, CURB-65, and SCAP score, in predicting the outcomes of patients with CAP and HCAP. We find no significant differences regarding the cumulative incidence of adverse events (death, septic shock, and need of transfer into an ICU) in patients included in the severe classes of the three scores. Cox regression analysis confirms that inclusion in severe classes is a factor significantly associated with death or development of complications. However, in the low classes, the incidence of adverse events is significantly higher in HCAP patients than in CAP ones. Thus, the three severity rules fail to detect a considerable amount of HCAP cases at increased risk of complicated outcome.

Several studies suggest that HCAP should be considered as a single clinical entity, different from CAP in terms of clinical presentation and outcome [1-3]. Our HCAP patients have a worse prognosis as compared to CAP despite no significant differences in terms of mean age or comorbid conditions between the two groups [1]. The poor prognosis of HCAP patients is related to a greater severity of disease (demonstrated by higher mean SOFA scores and by more frequent bilateral and multilobar lung involvement), and to the receipt of an initial antibiotic treatment not recommended by international guidelines [1]. These findings confirm previous studies alerting physicians to the greater likelihood of HCAP patients to receive inappropriate initial antibiotic treatment and their greater risk of in-hospital mortality [10]. Compared to CAP patients, those with HCAP are reported as frequently infected by MDR pathogens [2, 11]. This finding has been recently confirmed by a sub-analysis of our published prospective study, which shows a high frequency of $S$. aureus etiology in the HCAP group (the rate of methicillin resistance is $63.6 \%$ ) while S. pneumoniae predominates in the CAP group [12]. Thus, a critical factor influencing the in-hospital mortality is the receipt of an initial inappropriate
Table 4 Factors associated with intra-hospital mortality as assessed by multivariate Cox regression analysis

Three models are showed using each of the predictive score systems; the first class was used as reference

Bold values are those statistically significant

\begin{tabular}{lccc}
\hline & Hazard ratio & $95 \%$ CI & $P$ \\
\hline CURB-65 class II & 3.1 & $1.1-8.8$ & $\mathbf{0 . 0 3}$ \\
CURB-65 class III & 3.7 & $1.3-10.8$ & $\mathbf{0 . 0 1}$ \\
Empirical therapy not adherent to guidelines & 11.1 & $3.2-37.8$ & $<\mathbf{0 . 0 0 1}$ \\
PSI class II & 2.9 & $1.4-11.7$ & 0.28 \\
PSI class III & 6.7 & $1.2-36.9$ & $\mathbf{0 . 0 3}$ \\
Empirical therapy not adherent to guidelines & 10.7 & $3.1-37.2$ & $<\mathbf{0 . 0 0 1}$ \\
SCAP class II & 1.7 & $0.5-5.5$ & 0.40 \\
SCAP class III & 3.4 & $0.9-12.2$ & 0.06 \\
Empirical therapy not adherent to guidelines & 11.2 & $3.2-39.5$ & $<\mathbf{0 . 0 0 1}$ \\
\hline
\end{tabular}


Table 5 Factors associated with complicated outcome (combined endpoint) as assessed by multivariate Cox regression analysis

Three models are showed using each of the predictive score systems; the first class was used as reference

Bold values are those statistically significant

\begin{tabular}{lllc}
\hline & Hazard ratio & $95 \%$ CI & $P$ \\
\hline CURB-65 class II & 2.9 & $1.6-7.2$ & $\mathbf{0 . 0 2}$ \\
CURB-65 class III & 4.7 & $1.9-11.1$ & $\mathbf{0 . 0 0 1}$ \\
Empirical therapy not adherent to guidelines & 5.3 & $2.3-12.1$ & $<\mathbf{0 . 0 0 1}$ \\
PSI class II & 2.0 & $0.5-7.6$ & 0.30 \\
PSI class III & 4.1 & $1.4-11.7$ & $\mathbf{0 . 0 0 9}$ \\
Empirical therapy not adherent to guidelines & 5.3 & $2.3-12.3$ & $<\mathbf{0 . 0 0 1}$ \\
SCAP class II & 1.5 & $0.6-3.8$ & 0.40 \\
SCAP class III & 4 & $1.3-12.3$ & $\mathbf{0 . 0 1}$ \\
Empirical therapy not adherent to guidelines & 5.4 & $2.3-12.6$ & $<\mathbf{0 . 0 0 1}$ \\
\hline
\end{tabular}

Medicina Interna 5, Azienda Ospedaliera San CamilloForlanini, Roma: Laura Gasbarrone, Cecilia Serafini.

Clinica Medicina Interna 3, Azienda Ospedaliera Universitaria San Martino, Genova: Riccardo Ghio, Gabriele Zoppoli.

Medicina Generale III, Ospedale Luigi Sacco, Milano: Michele Cortellaro, Marina Magenta.

Medicina Generale I, Azienda Ospedaliera Universita degli Studi di Siena: Ranuccio Nuti, Roberto Valenti.

Presidio Ospedaliero C. Ondoli, Angera: Vincenzo Milano.

Medicina Interna, Azienda Ospedaliera San Giovanni Addolorata, Roma: Camillo Brandimarte, Paolo Carfagna.

Divisione Medicina Interna e Specialistica, Policlinico Universitario, Palermo: Riccardo Di Sciacca, Antonino Tuttolomondo.

Unità Operativa Medicina, Azienda Ospedaliera Card. G. Panico, Tricase: Maria Grazia Serra.

Semeiotica Medica, Policlinico S. Orsola Malpighi, Bologna: Mauro Bernardi, Silvia Li Bassi.

Dipartimento Medicina Interna e Gastroenterologia, Policlinico S. Orsola Malpighi, Bologna: Vincenzo Stanghellini, Elena Boschi.

Medicina Interna Universitaria "Cesare Frugoni", Policlinico, Bari: Salvatore Antonaci, Francesco Vella.

Dipartimento di Medicina Interna-Policlinico Universitario "G. Martino" di Messina: Antonino Catalano.

Divisione Medicina, Azienda Ospedaliera Universitaria, Modena: Maria Luisa Zeneroli, Elisabetta Ascari, Alberto Veggetti.

Ospedale Del Delta, Lagosanto: Roberto Manfredini, Susanna Gamberoni.

Istituto di Clinica Medica Generale, Ospedale di Cattinara, Trieste: Gianfranco Guarnieri, Angela Fioretto.

Medicina Interna, Ospedale "G. Mazzini” ASL Teramo: Dario Di Michele, Domenico Parisi.

Divisione di Medicina Interna, Ospedale Civile, Casorate Primo: Nicola Lucio Liberato, Esio Ronchi.

Medicina Interna, Ospedale Civile, Senigallia: Simonetta Sturbini, Paolo Canafoglia. 
Medicina Interna Ospedaliera, Azienda Ospedaliera Universitaria Sant'Anna, Ferrara: Massimo Gallerani, Benedetta Boari.

Medicina ad Alta Rotazione, Azienda Ospedaliera Universitaria Sant'Anna, Ferrara: Ingrid Nielsen.

Geriatria, Azienda Ospedaliera San Gerardo, Monza: Giorgio Annoni, Anna Rossetti.

Azienda Ospedaliera Ospedale di Circolo Fondazione Macchi, Varese: Matteo Bernasconi.

Medicina Interna, Azienda Ospedaliera Policlinico Bari: Carmela Giannatempo.

Medicina I, Azienda Ospedaliera G. Salvini, Garbagnate Milanese: Roberta Turconi, Maurizio Colombo.

Medicina Interna 2, Policlinico Ospedale Maggiore, Milano: Alberto Tedeschi, Raffaella Rossi.

Medicina Interna II, Policlinico S. Maria alle Scotte, Siena: Roberto Cappelli, Valentina Guidi.

Medicina 1 A.S.L. 2 Savonese Ospedale San Paolo Savona: Rodolfo Tassara, D. De Melis.

Medicina Interna, Fondazione IRCCS Ospedale Maggiore Policlinico, Mangiagalli e Regina Elena, Milano: Roberto Cosentini, Margherita Arioli, Francesco Salerno, Giulia Gobbo.

Medicina Generale, A.S.L. 17, Ospedale di Este: Fabio Presotto, Sergio Gallana.

Clinica Medica III, Fondazione IRCCS Policlinico San Matteo-Università di Pavia: Carlo Balduini, Giampiero Bertolino.

Medicina Interna, Presidio Ospedaliero-A.S.L. BA/5, Monopoli-Conversano: Giacomo Fera.

Clinica Medica I, Policlinico San Matteo, Pavia: Gino

Roberto Corazza, Ida Capriglione.

Medicina, Presidio Sanitario Gradenigo, Torino: Giulia Pilerio.

Dipartimento di Medicina Interna-Fondazione IRCCS Ospedale Maggiore Policlinico, Mangiagalli e Regina Elena \& Università degli studi di Milano: Maria Domenica Cappellini, Giovanna Fabio, Maria Carrabba.

Medicina Generale, Ospedale E. Bassini, Cinisello Balsamo, Azienda Ospedaliera S.Gerardo-Monza: Sheng Chin Wu, Maria Beatrice Secchi.

Medicina, Casa di Cura La Madonnina, Bari: Michele Leone.

Medicina Interna, Presidio Ospedaliero, Pescara: Lucrezia De Feudis.

Medicina Interna Ospedale San Salvatore Pesaro: Massimo Gunelli, Orazio Ferri.

Medicina Interna 2, Presidio Ospedaliero Cà Fondello, Treviso: Carlo Doroldi, Roberta Pistis.

Università Vita-Salute San Raffaele-Milano: Maria Grazia Sabbadini, Moreno Tresoldi.

Medicina Interna 5, Ospedale Unico della Versilia, Lido di Camaiore: Paola Lambelet, Stefano Fascetti.
Struttura Complessa di Medicina Interna, A.O. Ospedale di Lecco, P.O. di Merate: Massimo Vanoli, Gianluca Casella.

Clinica Medica Generale, Azienda Ospedali Civili, Brescia: Enrico Agabiti Rosei, Andrea Salvi.

Medicina Interna, Ospedale Civile dell'Annunziata, Cosenza: Alfonso Noto.

Medicina Interna, Ospedale San Giovanni DecollatoAndosilla, Civita Castellana: Antonio Perciaccante.

Medicina Interna, Ospedale Madre G. Vannini, Roma: Claudio Santini, Maurizia Galiè.

Istituto di Medicina Interna e Geriatria, Università Cattolica del Sacro Cuore, Policlinico A. Gemelli, Roma:

Giovanni Gasbarrini, Antonio Grieco.

Medicina 1, Azienda Ospedaliera di Busto Arsizio (VA)

Ospedale di Circolo di Busto Arsizio: Barbara Nardi.

Clinica Medica 1, Azienda Ospedaliera, Padova: Aldo

Gaetano Baritussio, Roberto Vannuccini.

Medicina Generale, Alessandria: Mauro Cappelletti.

Medicina d'Urgenza e Pronto Soccorso, Università Cattolica del Sacro Cuore, Policlinico A. Gemelli, Roma: Nicolò Gentiloni-Silveri.

Medicina Interna C, Policlinico G.B. Rossi, Verona: Alessandro Lechi, Germana Montesi.

\section{References}

1. Venditti M, Falcone M, Corrao S et al (2009) Comparison of the outcomes of patients hospitalized with community-acquired, health care-associated, and hospital-acquired pneumonia. Ann Intern Med 150:19-26

2. Zilberberg MD, Shorr AF, Micek ST et al (2008) Antimicrobial therapy escalation and hospital mortality among patients with health-care-associated pneumonia: a single-center experience. Chest 134:963-968

3. Shorr AF, Zilberberg MD, Micek ST, Kollef MH (2008) Prediction of infection due to antibiotic-resistant bacteria by select risk factors for health care-associated pneumonia. Arch Intern Med 168:2205-2210

4. Fine MJ, Auble TE, Yealy DM et al (1997) A prediction rule to identify low-risk patients with community-acquired pneumonia. N Engl J Med 336:243-250

5. Lim WS, van der Eerden MM, Laing R et al (2003) Defining community acquired pneumonia severity on presentation to hospital: an international derivation and validation study. Thorax 58:377-382

6. Espana PP, Capelastegui A, Gorordo I et al (2006) Development and validation of a clinical prediction rule for severe communityacquired pneumonia. Am J Respir Crit Care Med 174:1249-1256

7. Shindo Y, Sato S, Maruyama E et al (2008) Comparison of severity scoring systems A-DROP and CURB-65 for communityacquired pneumonia. Respirology 13:731-735

8. Mandell LA, Wunderink RG, Anzueto A et al (2007) Infectious Diseases Society of America. Infectious Diseases Society of America/American Thoracic Society consensus guidelines on the management of community-acquired pneumonia in adults. Clin Infect Dis 44(Suppl 2):S27-S72 
9. American Thoracic Society, Infectious Diseases Society of America (2005) Guidelines for the management of adults with hospital-acquired, ventilator-associated, and healthcare-associated pneumonia. Am J Respir Crit Care Med 171:388-416

10. Micek ST, Kollef KE, Reichley RM et al (2007) Health careassociated pneumonia and community-acquired pneumonia: a single-center experience. Antimicrob Agents Chemother $51: 3568-3573$
11. Shindo Y, Sato S, Maruyama E et al (2009) Health-care-associated pneumonia among hospitalized patients in a Japanese community hospital. Chest 135:633-640

12. Falcone M, Venditti M, Corrao S, Serra P for the SIMI (2011) Role of multidrug-resistant pathogens in health care-associated pneumonia. Lancet Infect Dis 11:12-13 